$5-1-2008$

\title{
The Propagation of Non-Lefschetz Type, The Gottlieb Group and Related Questions
}

John Oprea

Cleveland State University, J.OPREA@csuohio.edu

Follow this and additional works at: https://engagedscholarship.csuohio.edu/scimath_facpub

Part of the Mathematics Commons

How does access to this work benefit you? Let us know!

Publisher's Statement

The final publication is available at Springer via http://dx.doi.org/10.1007/s11784-008-0063-8

\section{Repository Citation}

Oprea, John, "The Propagation of Non-Lefschetz Type, The Gottlieb Group and Related Questions" (2008). Mathematics Faculty Publications. 239.

https://engagedscholarship.csuohio.edu/scimath_facpub/239

This Article is brought to you for free and open access by the Mathematics and Statistics Department at EngagedScholarship@CSU. It has been accepted for inclusion in Mathematics Faculty Publications by an authorized administrator of EngagedScholarship@CSU. For more information, please contact library.es@csuohio.edu. 


\title{
The propagation of non-Lefschetz type, the Gottlieb group and related questions
}

\author{
John Oprea \\ To Vladimir Arnold, who taught me symplectic geometry without knowing it

\begin{abstract}
This is a brief note which indicates how the property of being nonLefschetz may be propagated by equivariant symplectic maps. We also discuss some questions related to the Gottlieb group and nilpotency of symplectic manifolds.
\end{abstract}

Mathematics Subject Classification (2000). 55P99, 53D99.

I was first introduced to the subject of symplectic geometry in a seminar held at Ohio State University where the main text was Vladimir Arnold's Mathematical Methods in Classical Mechanics [Arn]. Although the cohomological nature of the symplectic form was even apparent then, I never dreamed that I would be led to work on the homotopy theory of symplectic geometry (see [LO1, LO2]). Homotopical methods have proved to be important in symplectic geometry and, in particular, when mixed with hard analysis, have led to a proof of Arnold's conjecture on fixed points of hamiltonian diffeomorphisms (see [RO], and [CLOT]). In this sense, Arnold's famous problems have been good for homotopy theory as well as geometry!

In this note, we consider the relationship between the existence of symplectic mappings between symplectic manifolds with circle action (or with appropriate Gottlieb structure) and the existence of Kähler structures (or, more generally, Lefschetz type structures of cohomology) on the manifolds. Much work has been done in recent years that clarifies the relationship between Lefschetz type and rational homotopy properties such as formality (see for instance, [Cav], [HT] and the forthcoming $[\mathrm{FOT}])$. Also, more work has been done on symplectically aspherical manifolds and their fundamental groups (see [IKRT]), and this leads to several interesting questions concerning Gottlieb groups and symplectic manifolds. 
The main results of this short note follow from the framework created in [LO2] for the study of symplectic circle actions on symplectic manifolds. With this in mind, we now turn to a brief review of that framework.

\section{Review of the $\lambda_{\hat{\alpha}}$-invariant}

An $S^{1}$-action and its orbit map may be generalized to produce certain basic elements in the fundamental group of $M$ and of the function space $\left(M^{M}, 1_{M}\right)$ in the following manner. Say that an element $\alpha \in \pi_{1}(M)$ is in the Gottlieb group of $M$, denoted $\alpha \in G(M)$, if there is an associated map

$$
A: S^{1} \times M \rightarrow M
$$

such that $\left.A\right|_{M}=1_{M}$ and $\left.A\right|_{S^{1}}=\alpha$. From [Go1] and [Go2], we know certain things about $G(M)$. In particular, we know that

(1) $G(M) \subseteq \mathcal{Z}\left(\pi_{1}(M)\right)$.

(2) If $M=K(\pi, 1)$, then $G(M)=\mathcal{Z}\left(\pi_{1}(M)\right)$.

By the exponential law, there is a map $\hat{\alpha}: S^{1} \rightarrow M^{M}, \hat{\alpha}(s)(x)=A(s, x)$, such that evaluation $\operatorname{ev}(f)=f(p)$ of a function at a basepoint $m \in M$ satisfies ev $\circ \hat{\alpha}=\alpha$. Hence, $\operatorname{ev}_{\#}(\hat{\alpha})=\alpha$ where ev $: \pi_{1}\left(M^{M}, 1_{M}\right) \rightarrow \pi_{1}(M)$. Note that a group action $A: S^{1} \times M \rightarrow M$ provides a Gottlieb element $\left.\alpha \simeq A\right|_{S^{1}}$ which may be identified with the homotopy class of the orbit map $a: S^{1} \rightarrow M$. Also note that it is possible for $\alpha$ to be nullhomotopic, but $\hat{\alpha}$ to be essential. For this reason, we shall be more interested in the associated map $A$ and its adjoint $\hat{\alpha}$ than in the (possible) Gottlieb element $\alpha$.

There is a classifying space $\operatorname{Baut}(M)$ for fibrations with fibre $M$ which obeys $\pi_{i+1} \operatorname{Baut}(M)=\pi_{i}\left(M^{M}, 1_{M}\right)$. Therefore, $\hat{\alpha} \in \pi_{1}\left(M^{M}, 1_{M}\right)$ corresponds to an element in $\pi_{2}$ Baut $(M)$ represented by a map $S^{2} \rightarrow$ Baut $(M)$. Pulling back the universal fibration with fibre $M$ gives a fibration

$$
M \stackrel{i}{\rightarrow} E \rightarrow S^{2}
$$

with $\alpha=\partial_{\#}(1) \in \operatorname{Im}\left(\partial_{\#}: \pi_{2} S^{2} \rightarrow \pi_{1} M\right)$. Such a fibration has a Wang sequence associated to it,

$$
\cdots \rightarrow H^{q}(E) \stackrel{i^{*}}{\rightarrow} H^{q}(M) \stackrel{\lambda_{\hat{\alpha}}}{\rightarrow} H^{q-1}(M) \rightarrow H^{q+1}(E) \rightarrow \cdots .
$$

The map $\lambda_{\hat{\alpha}}$, called the Wang derivation, is a derivation on $H^{*}(M)$. That is, $\lambda_{\hat{\alpha}}$ satisfies the relation $\left.\lambda_{\hat{\alpha}}(u v)=\lambda_{\hat{\alpha}}(u) v+(-1)^{|u|} u \lambda_{\hat{\alpha}}(v)\right)$. There is a beautiful connection between the Wang sequence and the map $A$. Namely, for any $u \in$ $H^{q}(M)$,

$$
A^{*}(u)=1 \times u+\bar{\sigma} \times \lambda_{\hat{\alpha}}(u),
$$


where $\bar{\sigma} \in H^{1}\left(S^{1}\right)$ is a chosen fixed generator, $\alpha=\mathrm{ev}_{\#}(\hat{\alpha})$ and $\times$ is the external product. In case $u \in H^{1}(M)$, we have $\alpha^{*}(u)=\lambda_{\hat{\alpha}}(u) \bar{\sigma}$, with $\lambda_{\hat{\alpha}}(u) \in H^{0}(M)=\mathbb{Q}$, and the expression $(\dagger)$ may be rewritten

$$
A^{*}(u)=1 \times u+\bar{\sigma} \times \lambda_{\hat{\alpha}}(u)=1 \times u+\alpha^{*}(u) \times 1 .
$$

For further details on the derivation $\lambda_{\hat{\alpha}}$, see [Go3]. We have a basic result, which follows immediately from the exactness of the Wang sequence.

Proposition 1.1. $\lambda_{\hat{\alpha}}(\omega)=0$ if and only if there exists $\bar{\omega} \in H^{2}(E)$ with $i^{*} \bar{\omega}=\omega$.

The following fundamental result connects the $\lambda_{\hat{\alpha}}$-invariant to symplectic geometry [LO2]:

Theorem 1.2. If $\hat{\alpha}$ comes from a symplectic $S^{1}$-action on a compact symplectic manifold $(M, \omega)$, then

$$
\lambda_{\hat{\alpha}}(\omega)=[i(\underline{X}) \omega],
$$

where $\underline{X}$ denotes the fundamental vector field associated to the symplectic $S^{1}$-action and $i(-)$ is interior multiplication.

Because the equality $[i(\underline{X}) \omega]=0$ is precisely what symplectic geometers mean by a Hamiltonian action, this theorem provides the basis for

Definition 1.3. A closed manifold $M^{2 n}$ is cohomologically symplectic (often shortened to $c$-symplectic) if there exists $\omega \in H^{2}(M)$ with $\omega^{n} \neq 0$. For a c-symplectic manifold $(M, \omega)$, an element $\hat{\alpha} \in \pi_{1}\left(M^{M}, 1_{M}\right)$ is said to be cohomologically Hamiltonian, or c-Hamiltonian, if $\lambda_{\hat{\alpha}}(\omega)=0$. If $\hat{\alpha}$ comes from an $S^{1}$-action and $\lambda_{\hat{\alpha}}(\omega)=0$, then we say that the action is c-Hamiltonian.

For the moment, let $M$ be an $N$-dimensional manifold, with top class $\mu \in$ $H^{N}(M ; \mathbb{Q})$. For applications we will specialize to a c-symplectic manifold $\left(M^{2 n}, \omega\right)$ with $\mu=\omega^{n}$. In general, we take $\hat{\alpha} \in \pi_{1}\left(M^{M}, 1_{M}\right)$ and $\alpha=\operatorname{ev}_{\#}(\hat{\alpha}) \in \pi_{1}(M)$. Recall that the Hurewicz homomorphism $h: \pi_{1}(M) \rightarrow H_{1}(M)$ is defined by $h(\gamma)=$ $\gamma_{*}(\sigma)$, where $\sigma$ is a generator of $H_{1}\left(S^{1} ; \mathbb{Z}\right)$ dual to $\bar{\sigma} \in H^{1}\left(S^{1} ; \mathbb{Z}\right)$ and $\gamma_{*}$ is the map on homology induced by $\gamma: S^{1} \rightarrow M$.

Our initial goal is to clarify the relationship between $\lambda_{\hat{\alpha}}$ and $h(\alpha)$. First, recall that Poincaré duality may be characterized in terms of cup, cap and Kronecker products: For $a \in H_{q}(M), z \in H_{N}(M)$ a fundamental class Kronecker dual to the top class $\mu$, the element $\hat{a} \in H^{N-q}(M)$ is Poincaré dual to $a$ if

$$
\langle b \cup \hat{a}, z\rangle=\langle b, \hat{a} \cap z\rangle=\langle b, a\rangle
$$

for any $b \in H^{q}(M)$. If $u \in H^{1}(M)$, then $u \cup \mu=0$ by dimensional considerations. Since $\lambda_{\hat{\alpha}}$ is a derivation, we get

$$
0=\lambda_{\hat{\alpha}}(u \cup \mu)=\lambda_{\hat{\alpha}}(u) \cdot \mu-u \cup \lambda_{\hat{\alpha}}(\mu) .
$$


Since $u$ is in degree 1 , we have $\alpha^{*}(u)=\lambda_{\hat{\alpha}}(u) \cdot \bar{\sigma}$. Hence,

$$
\left\langle u \cup \lambda_{\hat{\alpha}}(\mu), z\right\rangle=\left\langle\lambda_{\hat{\alpha}}(u) \cdot \mu, z\right\rangle=\lambda_{\hat{\alpha}}(u)=\left\langle\alpha^{*}(u), \sigma\right\rangle=\left\langle u, \alpha_{*}(\sigma)\right\rangle=\langle u, h(\alpha)\rangle .
$$

Our first result follows immediately from these observations.

Lemma 1.4. Let $\mu \in H^{N}(M)$ be a top class and let $\hat{\alpha} \in \pi_{1}\left(M^{M}, 1_{M}\right)$ with $\alpha=$ $\mathrm{ev}_{\#}(\hat{\alpha})$.

(1) For $u \in H^{1}(M), \lambda_{\hat{\alpha}}(u)=\langle u, h(\alpha)\rangle$.

(2) $\lambda_{\hat{\alpha}}(\mu)$ is Poincaré dual to $h(\alpha)$.

Combining Lemma 1.4 with another general observation yields the following result.

Proposition 1.5. Let $\mu \in H^{N}(M)$ be a top class and let $\hat{\alpha} \in \pi_{1}\left(M^{M}, 1_{M}\right)$ with $\alpha=\operatorname{ev}_{\#}(\hat{\alpha})$. We have the equivalences

$$
\lambda_{\hat{\alpha}}(\mu)=0 \Leftrightarrow \lambda_{\hat{\alpha}}\left(H^{1}(M)\right)=0 \Leftrightarrow h(\alpha)=0 .
$$

Proof. The first equivalence follows from a general result: If $\theta$ is a degree -1 derivation on a Poincaré duality algebra $H$, of top dimension $N$, then $\theta\left(H^{N}\right)=0$ if and only if $\theta\left(H^{1}\right)=0$ (see [LO1, Lemma 3.3] or [FOT, Proposition 7.60]). The second equivalence follows from Lemma 1.4(1).

This then gives

Proposition 1.6. Let $\left(M^{2 n}, \omega\right)$ be a c-symplectic manifold, with top class $\omega^{n}$, and let $\hat{\alpha} \in \pi_{1}\left(M^{M}, 1_{M}\right)$ with $\alpha=\operatorname{ev}_{\#}(\hat{\alpha})$. If $\hat{\alpha}$ is c-Hamiltonian (i.e. if $\lambda_{\hat{\alpha}}(\omega)=0$ ), then the three equivalent conditions of Proposition 1.5 hold.

Proof. Since $\lambda_{\hat{\alpha}}$ is a derivation, we have $\lambda_{\hat{\alpha}}\left(\omega^{n}\right)=n \omega^{n-1} \lambda_{\hat{\alpha}}(\omega)=0$.

Definition 1.7. A c-symplectic manifold $\left(M^{2 n}, \omega\right)$ has Lefschetz type if the multiplication homomorphism $\omega^{n-1}: H^{1}(M) \rightarrow H^{2 n-1}(M)$ is an isomorphism.

The Lefschetz type hypothesis allows for the implication of Proposition 1.6 to be reversed.

Theorem 1.8. Let $\left(M^{2 n}, \omega\right)$ be a c-symplectic manifold of Lefschetz type and let $\hat{\alpha} \in \pi_{1}\left(M^{M}, 1_{M}\right)$ with $\alpha=\operatorname{ev}_{\#}(\hat{\alpha})$. Then the following are equivalent:

(1) The element $\hat{\alpha}$ is c-Hamiltonian (i.e. $\lambda_{\hat{\alpha}}(\omega)=0$ ).

(2) $\lambda_{\hat{\alpha}}\left(\omega^{n}\right)=0$.

(3) $\lambda_{\hat{\alpha}}\left(H^{1}(M)\right)=0$.

(4) $h(\alpha)=0$.

Proof. In light of Proposition 1.6, we need only show that $h(\alpha)=0$ implies $\lambda_{\hat{\alpha}}(\omega)=0$. Now, by Lemma 1.4, $h(\alpha)$ is Poincaré dual to $\lambda_{\hat{\alpha}}\left(\omega^{n}\right)=n \cdot \lambda_{\hat{\alpha}}(\omega) \cup \omega^{n-1}$. By duality, $h(\alpha)=0$ then implies $n \cdot \lambda_{\hat{\alpha}}(\omega) \cup \omega^{n-1}=0$ and, by the Lefschetz type hypothesis, this can only happen if $\lambda_{\hat{\alpha}}(\omega)=0$. 


\section{2. c-Symplectic maps and the $\lambda_{\hat{\alpha}}$-invariant}

In this section, let $\left(N^{2 n}, \omega_{N}\right)$ and $\left(M^{2 m}, \omega_{M}\right)$ be c-symplectic manifolds with $S^{1}$ actions

$$
A_{N}: S^{1} \times N \rightarrow N, \quad A_{M}: S^{1} \times M \rightarrow M .
$$

Let $f: N \rightarrow M$ be a based homotopy equivariant map which is c-symplectic. We shall always require our maps between c-symplectic manifolds to be basepoint preserving, and homotopy equivariance will refer to basepoint preserving homotopies. Homotopy equivariance is expressed by a homotopy commutative diagram

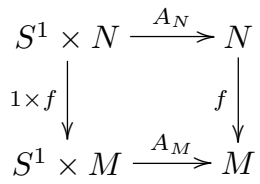

The map $f$ is c-symplectic if $f^{*}\left(\omega_{M}\right)=\omega_{N}$. Of course, any symplectic map is c-symplectic.

This diagram provides the link between $\lambda_{\hat{\alpha}_{N}}\left(\omega_{N}\right)$ and $\lambda_{\hat{\alpha}_{M}}\left(\omega_{M}\right)$.

Lemma 2.1. Let $\hat{\alpha}_{N} \in \pi_{1}\left(N^{N}, 1_{N}\right)$ and $\hat{\alpha}_{M} \in \pi_{1}\left(M^{M}, 1_{M}\right)$ be the adjoints of the respective actions $A_{N}$ and $A_{M}$. Then

$$
f^{*}\left(\lambda_{\hat{\alpha}_{M}}\left(\omega_{M}\right)\right)=\lambda_{\hat{\alpha}_{N}}\left(\omega_{N}\right) .
$$

Proof. We can compute two ways using homotopy equivariance:

$$
\begin{aligned}
(1 \times f)^{*} A_{M}^{*} \omega_{M} & =(1 \times f)^{*}\left(1 \times \omega_{M}+\bar{\sigma} \times \lambda_{\hat{\alpha}_{M}}\left(\omega_{M}\right)\right) \\
& =1 \times f^{*}\left(\omega_{M}\right)+\bar{\sigma} \times f^{*}\left(\lambda_{\hat{\alpha}_{M}}\left(\omega_{M}\right)\right) ; \\
A_{N}^{*} f^{*}\left(\omega_{M}\right) & =1 \times f^{*}\left(\omega_{M}\right)+\bar{\sigma} \times \lambda_{\hat{\alpha}_{N}}\left(f^{*}\left(\omega_{M}\right)\right) \\
& =1 \times f^{*}\left(\omega_{M}\right)+\bar{\sigma} \times \lambda_{\hat{\alpha}_{N}}\left(\omega_{N}\right) .
\end{aligned}
$$

By comparing the expressions, we see that $f^{*}\left(\lambda_{\hat{\alpha}_{M}}\left(\omega_{M}\right)\right)=\lambda_{\hat{\alpha}_{N}}\left(\omega_{N}\right)$.

Proposition 2.2. With the notations above, if $\hat{\alpha}_{M}$ is c-hamiltonian, then so is $\hat{\alpha}_{N}$.

Remark 2.3. Note that an equivariant map $f: N \rightarrow M$ satisfies the above. In particular, a symplectic map of symplectic manifolds which is equivariant with respect to $S^{1}$-actions fits the situation. Therefore, if the action on $M$ is Hamiltonian, then so is the action on $N$.

We can relate these ideas to the notion of Lefschetz type as follows.

Theorem 2.4. Let $f: N \rightarrow M$ be a c-symplectic map of c-symplectic manifolds $\left(N, \omega_{N}\right)$ and $\left(M, \omega_{M}\right)$ which is homotopy equivariant with respect to $S^{1}$-actions $A_{N}$ and $A_{M}$ respectively. For the adjoints $\hat{\alpha}_{N}$ of $A_{N}$ and $\hat{\alpha}_{M}$ of $A_{M}$, if $\hat{\alpha}_{N}$ satisfies the equivalent conditions of Proposition 1.5, but is not c-hamiltonian, then the same is true for $\hat{\alpha}_{M}$. Hence, $M$ cannot have Lefschetz type (and so, cannot be Kähler). 
Proof. Since $\hat{\alpha}_{N}$ satisfies the conditions of Proposition 1.5, the Hurewicz image $h\left(\alpha_{N}\right)$ is zero. Recall that $\alpha_{N}$ is represented by the composition

$$
S^{1 \stackrel{\hat{\alpha}_{N}}{\longrightarrow}} N^{N} \stackrel{\text { ev }}{\longrightarrow} N \text {. }
$$

The homotopy equivariance of the map $f$ provides a homotopy commutative diagram

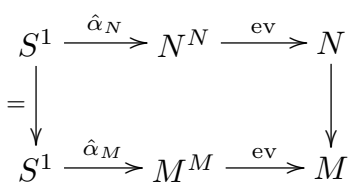

showing that $f_{\#}\left(\alpha_{N}\right)=\alpha_{M}$, where $f_{\#}: \pi_{1}(N) \rightarrow \pi_{1}(M)$. Then by the naturality of the Hurewicz map, we have

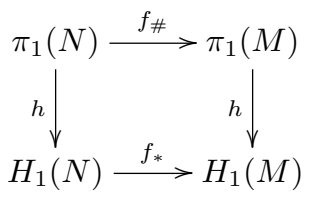

which gives $h\left(\alpha_{M}\right)=f_{*}\left(h\left(\alpha_{N}\right)\right)=0$ as well.

Now, $\hat{\alpha}_{N}$ is not c-hamiltonian by hypothesis, so $\lambda_{\hat{\alpha}_{N}}\left(\omega_{N}\right) \neq 0$. Then, by Lemma 2.1, $f^{*}\left(\lambda_{\hat{\alpha}_{M}}\left(\omega_{M}\right)\right)=\lambda_{\hat{\alpha}_{N}}\left(\omega_{N}\right)$, so $\lambda_{\hat{\alpha}_{M}}\left(\omega_{M}\right) \neq 0$ also. Therefore, $\hat{\alpha}_{M}$ satisfies the conditions of Proposition 1.5, but is not c-hamiltonian. By Theorem 1.8, $M$ cannot have Lefschetz type.

Remark 2.5. In fact, all that we have said above for $S^{1}$-actions also holds for maps $A: S^{1} \times N \rightarrow N$ which may not be actions, but which are adjoints of elements $\hat{\alpha}_{N} \in \pi_{1}\left(N^{N}, 1_{N}\right)$. Given such maps for $N$ and $M$ and a homotopy commutative diagram

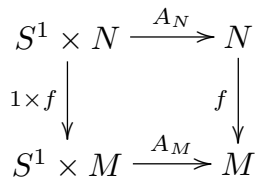

we can carry out the proofs of the results above with no changes. This will prove important in the next section.

\section{3. c-Symplectic maps of aspherical manifolds}

Let us apply the results above to the case where $\left(N, \omega_{N}\right)=\left(K(\pi, 1), \omega_{\pi}\right)$ and $\left(M, \omega_{M}\right)=\left(K(\rho, 1), \omega_{\rho}\right)$ are c-symplectic aspherical manifolds and $f: K(\pi, 1) \rightarrow$ $K(\rho, 1)$ is a c-symplectic map between them. Here, we can do without the necessity of requiring $S^{1}$-actions on the manifolds because it is a fact that any (effective) $S^{1}$ action $A$ on an aspherical manifold $K$ has an orbit map $\alpha: S^{1} \rightarrow K$ which induces 
an injection $\alpha_{\#}: \pi_{1}\left(S^{1}\right) \rightarrow \pi_{1}(K)$ whose image, in fact, must lie in the center of $\pi_{1}(K)$ (which is of course $G(K)$ ). Therefore, in the case of aspherical manifolds, we can turn our attention from $S^{1}$-actions to the centers of fundamental groups instead. Indeed, for an aspherical space $K$, it can be shown that

$$
\pi_{1}\left(K^{K}, 1_{K}\right) \stackrel{\mathrm{ev}}{\cong} \mathcal{Z}\left(\pi_{1}(K)\right)
$$

so that $\hat{\alpha}_{K}$ and $\alpha_{K}$ always correspond in this case. We shall assume this identification below when we show

Theorem 3.1. Let $\left(K(\pi, 1), \omega_{\pi}\right)$ and $\left(K(\rho, 1), \omega_{\rho}\right)$ be c-symplectic aspherical manifolds and let $f: K(\pi, 1) \rightarrow K(\rho, 1)$ be a c-symplectic map. Suppose there exist nontrivial $\alpha_{\pi} \in \mathcal{Z}(\pi)$ and $\alpha_{\rho} \in \mathcal{Z}(\rho)$ such that $f_{\#}\left(\alpha_{\pi}\right)=\alpha_{\rho}$. If $\alpha_{\pi}$ satisfies the equivalent conditions of Proposition 1.5, but is not c-hamiltonian, then the same is true for $\alpha_{\rho}$. Hence, $K(\rho, 1)$ cannot have Lefschetz type (and so, in particular, cannot be Kähler).

Proof. First notice that, because $\alpha_{\pi}$ is in the center of $\pi$, there is a homomorphism $\mathbb{Z} \times \pi \rightarrow \pi$ given by $A_{\pi \#}(n, g)=\alpha_{\pi}^{n} g$ and similarly for $\alpha_{\rho}$. We then have a commutative diagram

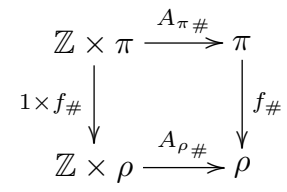

We can also make the computation

$$
\begin{aligned}
f_{\#} A_{\pi \#}(n, g) & =f_{\#}\left(\alpha_{\pi}^{n} g\right)=f_{\#}\left(\alpha_{\pi}^{n}\right) f_{\#}(g)=\alpha_{\rho}^{n} f_{\#}(g) \\
& =A_{\rho_{\#}}\left(n, f_{\#}(g)\right)=A_{\rho_{\#}}\left(1 \times f_{\#}\right)(n, g) .
\end{aligned}
$$

Because maps between aspherical spaces are classified at the fundamental group level and $\pi_{1}\left(S^{1}\right)=\mathbb{Z}$, this gives a homotopy commutative diagram

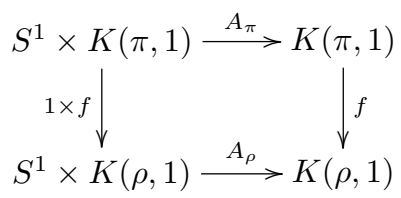

By Remark 2.5, we may now apply Theorem 2.4 to this situation to obtain the result.

It is known that nilmanifolds of Lefschetz type (e.g. Kähler nilmanifolds) are diffeomorphic to tori. The proof of this fact in [LO1, Theorem 3.1] (or in [O, Theorem 2.3.10]) may be interpreted as saying that, for a c-symplectic non-toral nilmanifold $K(\pi, 1)$, there always exists an element $\alpha_{\pi} \in \mathcal{Z}(\pi) \cap[\pi, \pi]$ such that $h\left(\alpha_{\pi}\right)=0$ (since $\alpha_{\pi} \in[\pi, \pi]$ and $H_{1}(\pi ; \mathbb{Z})=\pi /[\pi, \pi]$ ), but $\lambda_{\hat{\alpha}_{\pi}}\left(\omega_{\pi}\right) \neq 0$ (i.e. 
$\alpha_{\pi}$ is not c-hamiltonian). We can use this interpretation and the results above to propagate the non-Kählerness of nilmanifolds by c-symplectic maps as follows.

Corollary 3.2. Let $\left(K(\pi, 1), \omega_{\pi}\right)$ be a c-symplectic nilmanifold and suppose that $\left(K(\rho, 1), \omega_{\rho}\right)$ is a c-symplectic manifold such that $f: K(\pi, 1) \rightarrow K(\rho, 1)$ is a $c$ symplectic map. Suppose that $f_{\#}\left(\alpha_{\pi}\right)=\alpha_{\rho}$, where $\alpha_{\pi} \in \mathcal{Z}(\pi) \cap[\pi, \pi]$ is as above and $\alpha_{\rho} \in \mathcal{Z}(\rho)$ is nontrivial. If $K(\rho, 1)$ has Lefschetz type, then $K(\pi, 1)$ is a torus.

Proof. By Theorem 3.1, since $\alpha_{\pi}$ satisfies the conditions of Proposition 1.6, but is not c-hamiltonian, then the same holds for $\alpha_{\rho}$. This, however, contradicts Theorem 1.8 , since, by hypothesis, $K(\rho, 1)$ has Lefschetz type. Hence, the nilmanifold $K(\pi, 1)$ must be a torus since $\alpha_{\pi}$ always exists for non-toral nilmanifolds.

Remark 3.3. Corollary 3.2 would apply, in particular, to the case of a nilmanifold $N$ c-symplectically embedded as the fibre of a Mostow fibration

$$
N \rightarrow S \rightarrow T
$$

where $S$ is a solvmanifold and $T$ is a torus. If $\alpha_{\pi}$ may be extended to an element in the center of $\pi_{1}(S)$ and $S$ has Lefschetz type, then $N$ must be a torus. By our remarks above about $S^{1}$-actions on aspherical manifolds, such an extension would take place if the free $S^{1}$-action on $N$ given by $\alpha_{\pi}$ may be extended to all of $S$. Also note that, for completely solvable solvmanifolds, a symplectic form on $S$ may be chosen which restricts to one on $N([\mathrm{BG}])$.

\section{Some related questions}

R. Gompf showed that any finitely presented group can be realized as the fundamental group of certain symplectic 4-manifolds. More recently, in [IKRT] certain restrictions were found on the groups that can arise as fundamental groups of symplectic manifolds where the symplectic cohomology class annihilates the image of the Hurewicz homomorphism. These are the so-called symplectically aspherical manifolds. A fair amount is known about the homotopy theory of symplectically aspherical manifolds (see [LO2] as well as the reference [IKRT] mentioned above), but these manifolds are very special. In particular, these are the manifolds that are amenable to a homotopical proof of the Arnold conjecture on fixed points of hamiltonian diffeomorphisms (see, for instance, $[\mathrm{RO}, \mathrm{CLOT}]$ ). These are just first steps in understanding the homotopy theory of symplectic manifolds and certain classes of symplectic manifolds.

The first homotopical question that arises concerns the Gottlieb groups of symplectically aspherical manifolds. From [LO2, Theorem 4.12, Corollary 5.13], we know the following.

Theorem 4.1. If a circle acts on a symplectically aspherical manifold $M$, then the orbit map at a point, $S^{1} \rightarrow M$, induces an element of infinite order in the Gottlieb group $G(M)$. Further, if $M$ also has Lefschetz type, then the Hurewicz image of this Gottlieb element is of infinite order in $H_{1}(M ; \mathbb{Z})$. 
Here we see that homotopy theory and geometry of $M$ intertwine. In fact, however, this type of result is rare for Gottlieb groups. Indeed, it seems to be unknown if all finitely generated abelian groups arise as Gottlieb groups of compact manifolds. The result above, however, says that the following question may have an answer.

Question 4.2. What are the Gottlieb groups of symplectically aspherical manifolds?

A more fanciful line of thought asks

Question 4.3. What restrictions, if any, are placed on the Gottlieb groups of manifolds of Lefschetz type?

The Gottlieb group is also known to lie inside the subgroup of $\pi_{1}(M)$ consisting of all elements that act trivially on all higher homotopy groups (under the standard action). This brings up a general question about symplectic manifolds. Recall that a space $X$ is nilpotent if $\pi_{1}(X)$ acts nilpotently on $\pi_{j}(X)$ for all $j \geq 1$. The methods of homotopy theory and, especially, rational homotopy theory, work best for nilpotent spaces. So, if we are to make use of these methods, then it would be very nice to know the answer to the following

Question 4.4. How can nilpotent symplectic manifolds be recognized? If a symplectic manifold is a nilpotent space, what special homotopical properties are apparent? Conversely, what nilpotent spaces have symplectic or c-symplectic structures?

This is a question which connects geometry and homotopy theory in a fundamental way. Although we do not know of any general results in this direction, here is a result that gives a slight indication of how the action of the fundamental group on higher homotopy may be recognized in the symplectic world. While the proposition holds in general, it pays to think of $\omega$ as the symplectic (or c-symplectic) class. For a symplectic manifold $(M, \omega)$, the condition of symplectic asphericity is equivalent to the condition that $\omega=f^{*}(\widetilde{\omega})$, where $f: M \rightarrow K\left(\pi_{1}(M), 1\right)$ classifies the universal cover and $\widetilde{\omega}$ is some class in $H^{2}\left(K\left(\pi_{1}(M), 1\right) ; \mathbb{R}\right)$ (see [LO2]). Hence, $p^{*}(\omega)=0$, where $p: \widetilde{M} \rightarrow M$ is the universal cover. On the other hand, the result below applies to the generic case of symplectic manifolds that are not symplectically aspherical. The proposition is a special case of one found in [LMP], but the homotopical proof is new.

Proposition 4.5. Suppose that $M$ is a path connected space with a $\in H^{1}(M ; \mathbb{Q})$ and $\omega \in H^{2}(M ; \mathbb{Q})$ obeying $a \cup \omega=0$ and $p^{*}(\omega) \neq 0$, where $p: \widetilde{M} \rightarrow M$ is the universal cover. Then the action of $\pi_{1}(M)$ on $\pi_{2}(M)$ is nontrivial.

Proof. The proof and interpretation will come together in several steps.

Step 1. The condition $p^{*}(\omega) \neq 0$ is equivalent to saying that $\left.\omega\right|_{\pi_{2}(M)} \neq 0$, where $\omega \in H^{2}(M) \cong \operatorname{Hom}\left(H_{2}(M ; \mathbb{Z}), \mathbb{R}\right)$ is considered dual to homology and operating 
on the image of Hurewicz in $H_{2}(M ; \mathbb{Z})$. The equivalence of the conditions is implied immediately by the following commutative diagram:

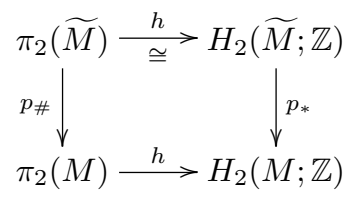

The condition $\left.\omega\right|_{\pi_{2}(M)} \neq 0$ removes the universal cover from consideration and focusses on $M$ and $\omega$.

Step 2. So, take $\gamma \in \pi_{2}(M)$ such that $\omega(h(\gamma)) \neq 0$ and $\alpha \in \pi_{1}(M)$ such that $a(h(\alpha)) \neq 0$. We wish to understand the action of $\alpha$ on $\gamma$ in a classical way. Namely, the deviation of the action from being trivial is detected by the Whitehead product

$$
\alpha \cdot \gamma-\gamma=[\alpha, \gamma]
$$

Thus, to show that the action of $\pi_{1}(M)$ on $\pi_{2}(M)$ is nontrivial, it is sufficient to show that the Whitehead product $[\alpha, \gamma]$ is nonzero. In order to do this, we will use Steenrod's functional cup product.

Step 3. Consider the following situation: $f: X \rightarrow Y$ and $v \in H^{q}(Y)$. We have a commutative diagram

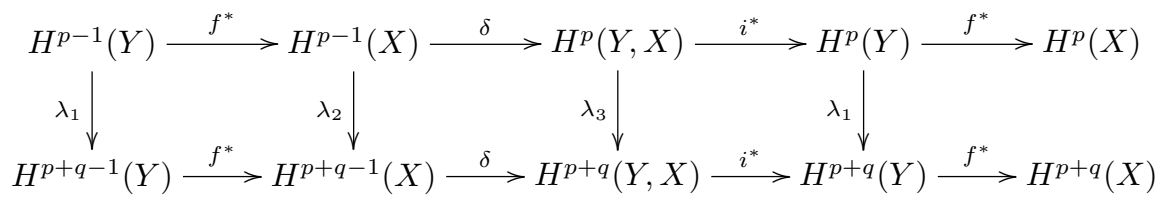

where $\lambda_{1}(y)=y \cup v, \lambda_{2}(u)=u \cup f^{*} v$ and $\lambda_{3}(z)=z \cup v$ (using relative cup product). Let $u \in \operatorname{Ker}\left(f^{*}\right) \cap \operatorname{Ker}\left(\lambda_{1}\right)$. Then $u \cup v=0$. Also, because $u \in \operatorname{Ker}\left(f^{*}\right)$, there exists $\beta \in H^{p}(Y, X)$ with $i^{*} \beta=u$ and

$$
\lambda_{1} i^{*} \beta=\lambda_{1} u=0=i^{*} \lambda_{3} \beta=i^{*}(\beta \cup v) .
$$

Hence, there exists $\tau \in H^{p+q-1}(X)$ with $\delta \tau=\beta \cup v$. Thus we can associate $\tau$ to the element $u$. This association $u \mapsto \tau$ provides a homomorphism

$$
\sigma: \operatorname{Ker}\left(f^{*}\right) \cap \operatorname{Ker}\left(\lambda_{1}\right) \rightarrow H^{p+q-1}(X) / f^{*} H^{p+q-1}(Y)+\lambda_{2} H^{p-1}(X)
$$

and we define the functional cup product (as a coset) by

$$
u \cup_{f} v:=\sigma(u) .
$$

The following facts are found in $[\mathrm{W}]$ :

- Fact 0. If $f$ is nullhomotopic, then $u \cup_{f} v=0$.

- Fact 1. If $f: X \rightarrow Y$ and $g: Y \rightarrow Z$, then $u \cup_{g f} v \subset g^{*}(u) \cup_{f} g^{*}(v)$ for $u, v \in H^{*}(Z)$. 
- Fact 2. For the Whitehead product map $\left[\iota_{p}, \iota_{q}\right]: S^{p+q-1} \rightarrow S^{p} \vee S^{q}$ and $u_{p}, u_{q}$ the respective generators of $H^{p}\left(S^{p}\right), H^{q}\left(S^{q}\right)$, we have

$$
u_{p} \cup_{\left[\iota_{p}, \iota_{q}\right]} u_{q}=-u_{p+q-1},
$$

where $u_{p+q-1}$ generates $H^{p+q-1}\left(S^{p+q-1}\right)$.

by

Now, for $\alpha \in \pi_{p}(X)$ and $\beta \in \pi_{q}(X)$, represent the Whitehead product $[\alpha, \beta]$

$$
F: S^{p+q-1} \stackrel{\left[\iota_{p}, \iota_{q}\right]}{\longrightarrow} S^{p} \vee S^{q} \stackrel{h}{\rightarrow} X
$$

where $\left.h\right|_{S^{p}}=\alpha,\left.h\right|_{S^{q}}=\beta$. Let $u, v \in H^{*}(X)$ be as above and such that $u \cup v=0$. Now, for $p, q>1, F\left(H^{p+q-1}(X)\right)=0$ since $F$ factors through $S^{p} \vee S^{q}$, and $F^{*} v=0$ since $H^{q}\left(S^{p+q-1}\right)=0$. For the case $p=1, q>1, F^{*}\left(H^{q}(X)\right)=0$ because the Whitehead product $\left[\iota_{p}, \iota_{q}\right]$ itself induces the zero homomorphism $H^{q}\left(S^{1} \vee S^{q}\right) \rightarrow$ $H^{q}\left(S^{q}\right)$ due to the fact that $\left[\iota_{p}, \iota_{q}\right]$ attaches the top cell in $S^{1} \times S^{q}$. Hence there is no indeterminacy in the definition of the functional cup product and we have

$$
u \cup_{F} v \in H^{p+q-1}\left(S^{p+q-1}\right) .
$$

By Fact 1 above, $u \cup_{F} v \subset h^{*}(u) \cup_{\left[\iota_{p}, \iota_{q}\right]} h^{*}(v)$.

Now suppose that $u \in H^{p}(X)$ is dual to a Hurewicz element $h(\alpha)$ for $\alpha \in$ $\pi_{p}(X)$ and $v \in H^{q}(X)$ is dual to a Hurewicz element $h(\beta)$ for $\beta \in \pi_{q}(X)$. Then $h^{*}(u)\left(\bar{u}_{p}\right)=u\left(h_{*}\left(\bar{u}_{p}\right)\right)=u(h(\alpha))=1$ and similarly $h^{*}(v)\left(\bar{u}_{q}\right)=1$. Thus,

$$
u \cup_{F} v=h^{*}(u) \cup_{\left[\iota_{p}, \iota_{q}\right]} h^{*}(v)=u_{p} \cup_{\left[\iota_{p}, \iota_{q}\right]} u_{q}=-u_{p+q-1}
$$

by Fact 2 . Since $u_{p+q-1} \neq 0$, by Fact $0, F=[\alpha, \beta] \neq 0$ as well.

Step 4. The situation above is almost exactly that of Proposition 4.5 with $\alpha \in$ $\pi_{1}(M)$ and $\beta=\gamma \in \pi_{2}(M)$. There is a slight difference which occurs because $\omega$ is not necessarily dual to $h(\gamma)$, but only is nonzero on it. This just introduces a nonzero factor $\xi$ into the equation

$$
a \cup_{[\alpha, \gamma]} \omega=\xi u_{2},
$$

where $u_{2}$ generates $H^{2}\left(S^{2}\right)$, and hence is nonzero. Therefore, the Whitehead product $[\alpha, \gamma]=\alpha \cdot \gamma-\gamma \neq 0$ and the action of $\pi_{1}(M)$ on $\pi_{2}(M)$ is nontrivial.

Another proof of Proposition 4.5 using the minimal models of rational homotopy theory is given in [FOT, Proposition 4.100]. Other interactions between symplectic (and complex) manifolds and rational homotopy theory may be found there as well.

\section{Self-maps of (symplectic) manifolds}

Another subject in symplectic geometry which has not been studied extensively is that of the homotopy theory of self-maps. Here is a type of result that relates a homotopical property of a space to the more geometrical question of how the space sits as a fibre in fibrations (or bundles). 
Proposition 5.1. Suppose $A$ is a manifold such that $H^{\text {odd }}(A ; \mathbb{Q})=0$ and $A$ sits as the fibre in a fibration $A \stackrel{i}{\rightarrow} E \rightarrow B$. If $f: A \rightarrow A$ is not rationally nullhomotopic, then $i \circ f: A \rightarrow E$ is also not rationally nullhomotopic.

Remark 5.2. By a result of [LO1], any symplectic (or c-symplectic) homogeneous space $A$ must be of maximal rank, and hence has $H^{\text {odd }}(A ; \mathbb{Q})=0$.

Proof of Proposition 5.1. If $i \circ f: A \rightarrow E$ were nullhomotopic, then $f$ would factor through the connecting map in the Puppe sequence, $\partial: \Omega B \rightarrow A$. Then Theorem 5.3 below would imply that $f$ is rationally null, a contradiction.

We shall now give a new proof of the fundamental theorem of Lupton and Smith. Their original proof used minimal models, but here we use only classical homotopy theory.

Theorem 5.3 ([LuSm, Theorem 3.2]). Let $f: A \rightarrow X$ be a map whose rationalization factors through an $H$-space. If $X$ is a finite complex and $H^{\text {odd }}(A ; \mathbb{Q})=0$, then $f$ is rationally trivial.

Since we are taking rationalizations in the theorem, from now on we will simply take $A$ and $X$ to be rational spaces. Also, by Hopf's theorem, we can take the $H$-space through which $f$ factors to be a product of $K(\mathbb{Q}, j)$ 's.

First, let us recall a standard result. Let $X_{n}$ denote the $n$-th Postnikov term of $X$. (Also, for later use, recall that the $n$-connective cover of $X$, denoted $X\langle n\rangle$, is the homotopy fibre of $X \rightarrow X_{n}$.) Milnor showed that, for any space $K$, there is a short exact sequence

$$
* \rightarrow \lim ^{1}\left[S K, X_{n}\right] \rightarrow[K, X] \rightarrow \underset{\lim }{\longleftarrow}\left[K, X_{n}\right] \rightarrow *,
$$

where $S K$ denotes suspension.

Lemma 5.4. If $X$ is a rational space, then $\varliminf^{1}\left[S K, X_{n}\right]=0$.

Proof. We always have $\left[S K, X_{n}\right]=\left[K, \Omega X_{n}\right]$ and, because of Hopf's result, we have the following homotopy commutative diagram expressing the fact that any map $K \rightarrow \Omega X_{n}$ can be lifted, up to homotopy, to $K \rightarrow \Omega X_{n+1}$.

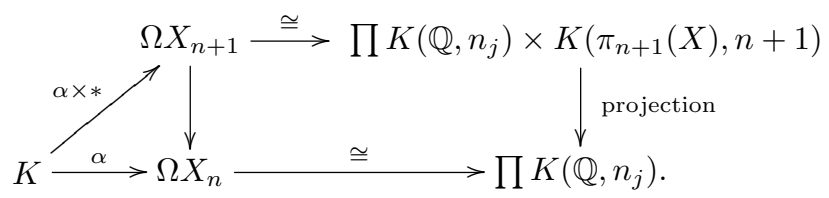

Therefore, we see that we have surjections of groups $\left[S K, X_{n+1}\right] \rightarrow\left[S K, X_{n}\right]$ for all $n$. The tower of these groups then satisfies the Mittag-Leffler condition, so $\lim ^{1}\left[S K, X_{n}\right]=0$.

The result above is sometimes expressed by saying that there are no rational phantom maps. Therefore, in order to understand the homotopy class of a rational map $K \rightarrow X$, it is sufficient to understand the projections to all Postnikov pieces. 
Now suppose we have a factorization $A \stackrel{g}{\rightarrow} H \stackrel{h}{\rightarrow} X$ with $f=h g$. Let $H=$ $H_{o} \times H_{e}$, where

$$
H_{o}=\prod K\left(\mathbb{Q}, 2 k_{j}+1\right) \quad \text { and } \quad H_{e}=\prod K\left(\mathbb{Q}, 2 k_{i}\right)
$$

by Hopf's theorem. It is always true that $[K, X \times Y]=[K, X] \times[K, Y]$, so the homotopy class of $g$ is determined by that of the projections $A \rightarrow H_{o}$ and $A \rightarrow H_{e}$. Because $H_{o}=\prod K\left(\mathbb{Q}, 2 k_{j}+1\right)$, the class of $A \rightarrow H_{o}$ is given by an element in $H^{\text {odd }}(A ; \mathbb{Q})=0$. Hence, we may restrict attention to $H_{e}$. In fact, we now focus on the restriction $h_{e}: H_{e} \rightarrow X$. Note that $H_{e}$ has the property that every element in $H^{*}\left(H_{e} ; \mathbb{Q}\right)$ has infinite height (i.e. cup product powers never vanish).

Lemma 5.5. Suppose $Z$ has the property that every element in $H^{*}(Z ; \mathbb{Q})$ has infinite height and let $X$ be a rational space of finite category (e.g. the rationalization of a finite complex). Then $[Z, X]=*$.

Proof. First note that $H^{\text {odd }}(Z ; \mathbb{Q})=0$ since elements of odd degree have finite height 1 . Secondly, note that a composition $Z \stackrel{h}{\rightarrow} X \stackrel{p_{2}}{\rightarrow} X_{2}=K\left(\pi_{2}(X), 2\right)$ must be nullhomotopic because the homotopy class is determined by an element of $H^{2}\left(Z ; \pi_{2}(X)\right)$ which is pulled back from an element of $H^{2}\left(X ; \pi_{2}(X)\right)$ by $h^{*}$. But the element in $H^{2}\left(X ; \pi_{2}(X)\right)$ would then have infinite height, and this is impossible by the assumption that $X$ has finite category (which means that the cuplength of $X$ is finite). This begins an induction where, for any map $h: Z \rightarrow X$, we have the following homotopy commutative diagram:

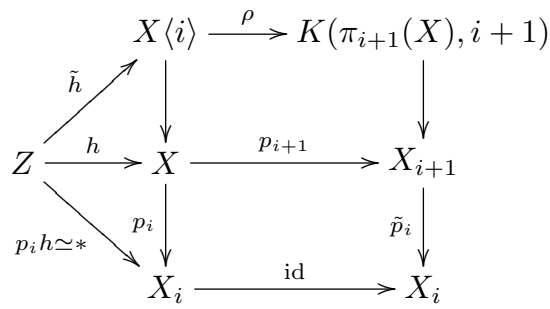

Of course, we are able to factor through the fibres only because the map $p_{i} h$ is trivial.

There are two cases. If $i+1$ is odd, then the homotopy class of $\rho \tilde{h}: Z \rightarrow$ $K\left(\pi_{i+1}(X), i+1\right)$ is an element of $H^{\text {odd }}(Z ; \mathbb{Q})=0$. Hence, the composition $p_{i+1} h: Z \rightarrow X_{i+1}$ is also trivial and the induction continues.

If $i+1$ is even, then, by what we have just said, we have a factorization through fibres $Z \stackrel{\tilde{h}}{\rightarrow} X\langle i\rangle \stackrel{\rho}{\rightarrow} K\left(\pi_{i+1}(X), i+1\right)$. As before, the class of this map is given by an element in $H^{i+1}\left(Z ; \pi_{i+1}(X)\right)$ which is pulled back from an element in $H^{i+1}\left(X\langle i\rangle ; \pi_{i+1}(X)\right)$ by $\tilde{h}^{*}$. If this map is not nullhomotopic, then this element in $H^{i+1}\left(X\langle i\rangle ; \pi_{i+1}(X)\right)$ would have infinite height. But this is impossible because the $i$-connective covering map $X\langle i\rangle \rightarrow X$ is injective on homotopy groups, and the 
Mapping Theorem of rational homotopy theory (see [FOT, Theorem 2.81]) then says that

$$
\operatorname{cup}_{0}(X\langle i\rangle) \leq \operatorname{cat}_{0}(X\langle i\rangle) \leq \operatorname{cat}_{0}(X)<\infty .
$$

Hence, $p_{i+1} h \simeq *$. Therefore, we see inductively that $p_{j} h \simeq *: Z \rightarrow X_{j}$ for all $j$. By Lemma 5.4, we have $h \simeq *$.

Proof of Theorem 5.3. We have already seen that we can restrict to a map $h_{e}$ : $H_{e} \rightarrow X$, where $H_{e}=\prod K\left(\mathbb{Q}, 2 k_{i}\right)$. But then $H_{e}$ satisfies the hypotheses of Lemma 5.5 , so $\left[H_{e}, X\right]=*$. Therefore, we obtain

$$
f \simeq h g \simeq h\left(g_{e} \times g_{o}\right) \simeq h\left(g_{e} \times *\right) \simeq h_{e} g_{e} \simeq * g_{e} \simeq *
$$

\section{References}

[Arn] V. I. Arnold, Mathematical Methods of Classical Mechanics (with contributions by K. Vogtmann and A. Weinstein). Grad. Texts in Math. 60, Springer, New York, 2000.

[BG] C. Benson and C. Gordon, Kähler structures on solvmanifolds for completely solvable Lie groups. Proc. Amer. Math. Soc. 108 (1990), 971-990.

[Cav] G. Cavalcanti, The Lefschetz property, formality and blowing up in symplectic geometry. Trans. Amer. Math. Soc. 359 (2007), 333-348.

[CLOT] O. Cornea, G. Lupton, J. Oprea and D. Tanré, Lusternik-Schnirelmann Category. Math. Surveys Monogr. 103, Amer. Math. Soc., Providence, RI, 2003.

[FOT] Y. Félix, J. Oprea and D. Tanré, Algebraic Models in Geometry. Oxford Grad. Texts in Math. 17, Oxford Univ. Press, 2008.

[Go1] D. Gottlieb, A certain subgroup of the fundamental group. Amer. J. Math. 87 (1965), 840-856.

[Go2] D. Gottlieb, Evaluation subgroups of homotopy groups, Amer. J. Math. 91 (1969), 729-756.

[Go3] D. Gottlieb, Applications of bundle map theory. Trans. Amer. Math. Soc. 171 (1972), 23-50.

[HT] B. Hajduk and A. Tralle, Exotic smooth structures and symplectic forms on closed manifolds. Preprint, 2007.

[IKRT] R. Ibáñez, J. Kędra, Y. Rudyak, and A. Tralle, On fundamental groups of symplectically aspherical manifolds. Math. Z. 248 (2004), 805-826.

[LMP] F. Lalonde, D. McDuff and L. Polterovich, On the flux conjectures. In: Geometry, Topology, and Dynamics (Montreal, PQ, 1995), CRM Proc. Lecture Notes 15, Amer. Math. Soc., Providence, RI, 1998, 69-85.

[LO1] G. Lupton and J. Oprea, Symplectic manifolds and formality. J. Pure Appl. Algebra 91 (1994), 193-207.

[LO2] G. Lupton and J. Oprea, Cohomologically symplectic spaces: toral actions and the Gottlieb group. Trans. Amer. Math. Soc. 347 (1995), 261-288.

[LuSm] G. Lupton and S. Smith, Cyclic maps in rational homotopy theory, Math. Z. 249 (2005), 113-124. 
[O] J. Oprea, Symplectic geometry and homotopy theory. In: Proc. Daewoo Workshop in Pure Math. 15, Part 3, Korean Academic Council, Chungju City, 1995, $1-41$.

[RO] Y. Rudyak and J. Oprea, On the Lusternik-Schnirelmann category of symplectic manifolds and the Arnold conjecture. Math. Z. 230 (1999), 673-678.

[W] G. Whitehead, Elements of Homotopy Theory. Grad. Texts in Math. 61, Springer, 1978.

Post-print standardized by MSL Academic Endeavors, the imprint of the Michael Schwartz Library at Cleveland State University, 2017 